\title{
Álvarez Díaz, J. A. (2018). Aspectos éticos de la nanotecnología en la atención de la salud. Ciudad de México: Universidad Autónoma Metropolitana
}

\section{Bárbara Rubio}

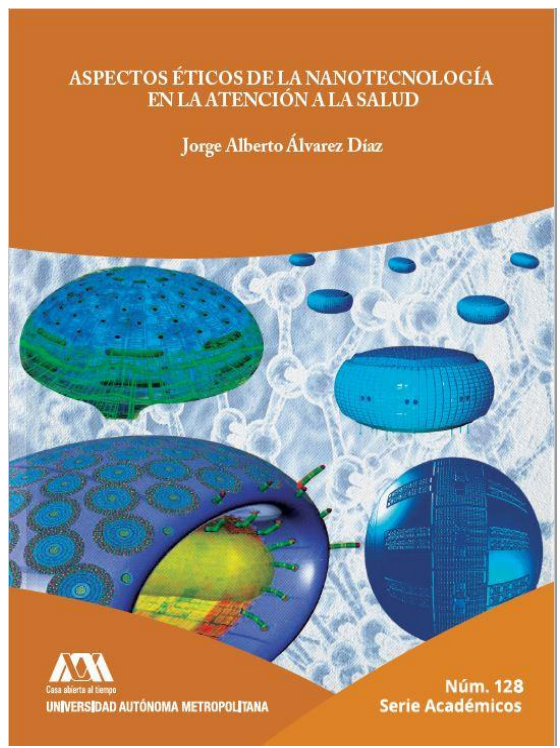

Ha pasado ya más de medio siglo desde que el premio nobel de Física Richard Feynman pronunciara en el Instituto de Tecnología de California su famoso discurso titulado "Hay mucho espacio en el fondo", que fue considerado por muchos el origen de la nanotecnología (There is Plenty of Room at the Bottom, 1959), aunque este término como tal fuera acuñado por Taniguchi en 1974.

Feynman describió la posibilidad de poder manipular las cosas "átomo por átomo", lo que permitiría trabajar con materiales a escala nanométrica (millonésima parte de un milímetro). Cuando se manipula la materia a escala tan minúscula presenta fenómenos y propiedades totalmente nuevas.

A partir de Feynman y posteriormente de Taniguchi, las aplicaciones y materiales que se manejan a nanoescala y hacen funcionar muchos de los aparatos que ya se utilizan, se han ido multiplicando de forma exponencial, marcando un antes y un después en múltiples especialidades científicas como la industria, la informática, la farmacología, el medioambiente y también la salud.

Respecto a las aplicaciones a la salud, a medida que se avanza en la comprensión y el control de la materia a nanoescala se abren nuevas posibilidades al operar la nanotecnología en la misma escala que la biología. La posibilidad de insertar sondas nanométricas en el interior de determinadas células y conocer su complejo funcionamiento permitiría detectar precozmente si se ha producido una alteración en la misma y poder actuar también precozmente. En el caso del cáncer, por ejemplo, tendría mucha repercusión en la detección precoz y tratamiento del mismo.

Muchos autores consideran la nanotecnología como "la tecnología transformadora del siglo XXI" y se ha convertido en la ciencia y la tecnología donde más se está 
invirtiendo en investigación y desarrollo a escala mundial. Pero al igual que ha ocurrido con otras revoluciones científicas, al mismo tiempo que las nanoaplicaciones tienen la capacidad de abarcar todos los ámbitos de la existencia humana e incluso planetaria, surgen muchos interrogantes a resolver y nuevos retos éticos que abordar.

La intención del autor Jorge Alberto Álvarez Díaz en este libro es proponer un marco para el análisis de los problemas éticos que surjan con la introducción de la nanotecnología en la atención a la salud.

Para ello, el autor, en la primera parte del libro, clarifica algunos conceptos básicos de la nanotecnología, necesarios para comprender por qué lo más pequeño, lo "nano", se convierte en algo cada vez más grande. Comienza explicando el prefijo "nano" para finalizar con su aplicación a las distintas áreas específicas de la nanotecnología. Cuando esta se aplica al campo de las ciencias de la vida se hablaría de una bionanotecnología o una nanobiotecnología, términos que en ocasiones se superponen.

Además, el autor hace un breve pero conciso recorrido histórico de la nanotecnología bajo el título "del neolítico al nanolítico". Para él, el enfoque histórico es fundamental para comprender las disciplinas científicas y filosóficas. Como diría el filósofo Ortega y Gasset: "Solo el sentido histórico, la admisión de la diferencia y la distancia entre culturas, permite avanzar en el esfuerzo de comprensión...". Aunque Álvarez Díaz centra el inicio y desarrollo posterior de la nanotecnología en la segunda mitad del siglo XX, principalmente en Feynman y Drexler, existe algún dato prehistórico como la copa de Licurgo Tracia. Se trata de una copa de vidrio dicroico procedente de Roma, que data del siglo IV a.C. y que se exhibe en el Museo Británico. Esta copa contenía partículas de oro (que hoy en día se sabe que son nanométricas) en suspensión coloidal que permitían cambiar el color de la copa de verde a rojo cuando pasaba el haz de luz a través de la copa.

En el mundo actual, la nanotecnología tiene infinidad de aplicaciones en muy diversas áreas (electrónica, informática, industria, medicina) y existen múltiples empresas distribuidas en más de una veintena de países dedicadas a explotar estas aplicaciones. El campo más avanzado y de mayor impacto se corresponde con el diseño de nuevos materiales con propiedades nuevas y mejoradas (más fuertes y más ligeros) como se pueden encontrar en artículos deportivos, cosméticos, adhesivos dentales etc. Es en esta nanotecnología del presente donde se empiezan a vislumbrar los problemas éticos. Problemas relacionados con la falta de información por parte de las empresas acerca de los riesgos y beneficios de los nanomateriales contenidos en sus productos (sus riesgos y beneficios), la falta de control por parte de las autoridades, aspectos éticos medioambientales, seguridad y privacidad, justicia en la asignación de recursos, ética de la investigación de nuevos productos.

En cuanto a la nanotecnología del futuro, si bien parece plantear problemas éticos importantes, como los que se contemplan en el programa de los transhumanistas que apoyan el empleo de las nuevas nanotecnologías hasta eventualmente poder eliminar la enfermedad e incluso la muerte, se perciben como de mayor urgencia los que se están planteando en el momento actual.

En la segunda sección del libro, el autor se centra en la aplicación de la nanobiotecnología a la salud, lo que se conoce como nanomedicina. Se ha visto que la 
capacidad para estructurar materiales y dispositivos a escala molecular puede aportar enormes beneficios a la práctica de la medicina. Las principales áreas de actuación de la nanomedicina son el diagnóstico (nanodiagnóstico) a través de herramientas que permiten visualizar nuestro organismo a escala molecular, como es el caso de los biosensores y también de las técnicas de imagen mediante la utilización de puntos cuánticos. En segundo lugar, el tratamiento de enfermedades, mediante dispositivos que permiten la administración dirigida de fármacos y su liberación controlada. Finalmente, la medicina regenerativa a partir de biomateriales e implantes inteligentes, moléculas de señalización bioactivas y terapias basadas en células. De gran interés resulta la posibilidad de trabajar con dispositivos que puedan realizar a la vez un diagnóstico y tratamiento individualizado (teranóstico), lo que daría lugar a una medicina personalizada.

Aunque la mayor parte de las aplicaciones de la nanomedicina se encuentran hoy en día en fase de investigación, a medida que se avanza en la comprensión y el control de la materia a la nanoescala, las posibilidades diagnósticas y terapéuticas de la nanomedicina se convertirán en una de las revoluciones científicas más importantes para la humanidad.

A lo largo de la historia el ser humano ha utilizado sus conocimientos para transformar la naturaleza y adaptarse al medio. Conforme ha ido evolucionando y avanzando en sus conocimientos, se ha ido dando cuenta de que la investigación científica y su aplicación no está exenta de riesgos y puede ocasionar daño. En la era de la nanociencia y la nanotecnología el ser humano no solo ha llegado a transformar la naturaleza para poder adaptarse a ella, sino que además ahora la puede manipular y adaptarla a sí mismo. Este nuevo "poder" en manos del ser humano trae consigo nuevos retos éticos que de alguna manera hay que abordar para buscar respuestas que sean prudentes y evitar los efectos nocivos que este nuevo poder pueda ocasionar.

En la tercera sección de esta obra, Álvarez Díaz propone un marco que permita realizar un análisis riguroso de los problemas éticos que surgen con la aplicación de las nanotecnologías en la atención a la salud. Argumenta que la nanociencia y nanotecnología plantean problemas éticos específicos que requieren soluciones específicas. La ética de las nanotecnologías (nanoética), por tanto, habría que entenderla como una nueva forma de ética aplicada. En busca de ese marco, el autor, con un enfoque "nano", dirige su mirada hacia la bioética y encuentra que la nanotecnología interacciona con los tres niveles de la bioética: micro (personal), meso (institucional) y macro-bioético (global), y aunque cada nivel presenta problemas distintos, están estrechamente articulados entre sí. Este enfoque podría ser el de una nanobioética que trataría los problemas éticos que surgen de la nanobiotecnología, donde se incluiría la nanomedicina.

Los problemas éticos que plantean las aplicaciones de la nanotecnología en la atención a la salud son numerosos. Ejemplos pueden ser la confidencialidad de los datos personales, la transparencia en la información sobre los posibles riesgos y la inequidad en el acceso a las nuevas posibilidades diagnósticas y terapéuticas, con el consecuente incremento en la brecha entre ricos y pobres. Para resolver estos y otros posibles conflictos éticos que plantea la nanobiotecnología, Álvarez Díaz propone y 
describe el método de la deliberación moral del Profesor Diego Gracia en orden a tomar decisiones prudentes (la más óptima posible en condiciones de incertidumbre).

No cabe duda de que las aplicaciones de la nanotecnología en la atención a la salud abarcan un amplio abanico de posibilidades con implicaciones éticas, sociales, ambientales y legales, lo que pone de manifiesto la necesidad de establecer unos mínimos éticos, entre ellos el principio de precaución.

Para contestar a preguntas como: ¿es éticamente admisible hacer todo lo científica y tecnológicamente posible?, sería necesario tomar conciencia de la necesidad de un debate deliberativo a todos los niveles, desde el local, pasando por el regional y nacional, hasta llegar a escala mundial (democracia deliberativa).

La lectura de Aspectos éticos de la nanotecnología en la atención a la salud, para alguien que hasta el momento no ha estado familiarizada con las tecnologías a escala "nano", no solo ha supuesto un despertar del interés y la curiosidad por la materia sino también motivo de inquietud y preocupación. La necesidad de hacer un llamamiento a la responsabilidad moral de cada individuo se hace imperativo, pues la responsabilidad derivada de su acción alcanza su propio entorno, y de este entorno depende su propia existencia. 